\title{
Stochastic solutions of nonlinear pde's: McKean versus superprocesses
}

\author{
R. Vilela Mendes \\ CMAF - Complexo Interdisciplinar, Universidade de Lisboa \\ (Av. Gama Pinto 2, 1649-003, Lisbon, vilela@cii.fc.ul.pt) \\ Instituto de Plasmas e Fusão Nuclear, IST \\ (Av. Rovisco Pais, 1049-001 Lisbon)
}

\begin{abstract}
Stochastic solutions not only provide new rigorous results for nonlinear pde's but also, through its local non-grid nature, are a natural tool for parallel computation.

There are two methods to construct stochastic solutions: the McKean method and superprocesses. Here a comparison is made of these two approaches and their strenghts and limitations are discussed.
\end{abstract}

\section{Introduction: Stochastic solutions and their uses}

A stochastic solution of a linear or nonlinear partial differential equation is a stochastic process which, when started from a particular point in the domain generates after a time $t$ a boundary measure which, when integrated over the initial condition at $t=0$, provides the solution at the point $x$ and time $t$. For example for the heat equation

$$
\partial_{t} u(t, x)=\frac{1}{2} \frac{\partial^{2}}{\partial x^{2}} u(t, x) \quad \text { with } \quad u(0, x)=f(x)
$$


the stochastic process is Brownian motion and the solution is

$$
u(t, x)=\mathbb{E}_{x} f\left(X_{t}\right)
$$

$\mathbb{E}_{x}$ meaning the expectation value, starting from $x$, of the process

$$
d X_{t}=d B_{t}
$$

The domain here is $\mathbb{R} \times[0, t)$ and the expectation value in (2) is indeed the inner product $\left\langle\mu_{t}, f\right\rangle$ of the initial condition $f$ with the measure $\mu_{t}$ generated by the Brownian motion at the $t$-boundary. The usual integral solution,

$$
u(t, x)=\frac{1}{2 \sqrt{\pi}} \int \frac{1}{\sqrt{t}} \exp \left(-\frac{(x-y)^{2}}{4 t}\right) f(y) d y
$$

with the heat kernel, has exactly the same interpretation. Of course, an important condition for the stochastic process (Brownian motion in this case) to be considered the solution of the equation is the fact that the same process works for any initial condition. This should be contrasted with stochastic processes constructed from particular solutions.

That the solutions of linear elliptic and parabolic equations, both with Cauchy and Dirichlet boundary conditions, have a probabilistic interpretation is a classical result and a standard tool in potential theory [1 [2 [3]. In contrast with the linear problems, explicit solutions in terms of elementary functions or integrals for nonlinear partial differential equations are only known in very particular cases. Therefore the construction of solutions through stochastic processes, for nonlinear equations, has become an active field in recent years. The first stochastic solution for a nonlinear pde was constructed by McKean [4] for the KPP equation. Later on, the exit measures provided by diffusion plus branching processes [5] 6] as well as the stochastic representations recently constructed for the Navier-Stokes [7] [8] [9] [10] [11], the Vlasov-Poisson [12] [13] [15], the Euler [14] and a fractional version of the KPP equation [16] define solution-independent processes for which the mean values of some functionals are solutions to these equations. Therefore, they are exact stochastic solutions.

In the stochastic solutions one deals with a process that starts from the point where the solution is to be found, a functional being then computed on the boundary or in some cases along the whole sample path. In addition to providing new exact results, the stochastic solutions are also a promising tool for numerical implementation. This is because: 
(i) Deterministic algorithms grow exponentially with the dimension $d$ of the space , roughly $N^{d}$ ( $\frac{L}{N}$ being the linear size of the grid). This implies that to have reasonable computing times, the number of grid points may not be sufficient to obtain a good local resolution for the solution. In contrast a stochastic simulation only grows with the dimension of the process, typically of order $d$.

(ii) In general, deterministic algorithms aim at obtaining the global behavior of the solution in the whole domain. That means that even if an efficient deterministic algorithm exists for the problem, a stochastic algorithm might still be competitive if only localized values of the solution are desired. This comes from the very nature of the stochastic representation processes that always start from a definite point of the domain. According to what is desired, real or Fourier space representations should be used. For example by studying only a few high Fourier modes one may obtain information on the small scale fluctuations that only a very fine grid would provide in a deterministic algorithm.

(iii) Each time a sample path of the process is implemented, it is independent from any other sample paths that are used to obtain the expectation value. Likewise, paths starting from different points are independent from each other. Therefore the stochastic algorithms are a natural choice for parallel and distributed implementation. Provided some differentiability conditions are satisfied, the process also handles equally well simple or complex boundary conditions.

(iv) Stochastic algorithms may also be used for domain decomposition purposes [17 [18] [19]. One may, for example, decompose the space in subdomains and then use in each one a deterministic algorithm with Dirichlet boundary conditions, the values on the boundaries being determined by a stochastic algorithm, thus minimizing the time-consuming communication problem between domains.

There are basically two methods to construct stochastic solutions. The first method, which will be called the McKean method, is essentially a probabilistic interpretation of the Picard series. The differential equation is written as an integral equation which is rearranged in a such a way that the coefficients of the successive terms in the Picard iteration obey a normalization condition. The Picard iteration is then interpreted as an evolution and branching process, the stochastic solution being equivalent to importance sampling of the normalized Picard series. The second method constructs the boundary measures of a measure-valued stochastic process (a superprocess) 
and obtain the solutions of the differential equation by a scaling procedure.

In this paper the two methods are compared and in the final sections one shows how the superprocess construction may be extended to larger classes of partial differential equations by going from process on measures to processes on signed measures and processes on distributions.

\section{McKean and superprocesses: The KPP equa- tion}

\subsection{The KPP equation: McKean's formulation}

To illustrate the two methods for the construction of stochastic solutions a classical example will be used, namely the KPP equation [4]

$$
\frac{\partial v}{\partial t}=\frac{1}{2} \frac{\partial^{2} v}{\partial x^{2}}+v^{2}-v
$$

with initial data $v(0, x)=g(x)$

Let $G(t, x)$ be the Green's operator for the heat equation $\partial_{t} v(t, x)=$ $\frac{1}{2} \frac{\partial^{2}}{\partial x^{2}} v(t, x)$

$$
G(t, x)=e^{\frac{1}{2} t \frac{\partial^{2}}{\partial x^{2}}}
$$

Then the equation in integral form is

$$
v(t, x)=e^{-t} G(t, x) g(x)+\int_{0}^{t} e^{-(t-s)} G(t-s, x) v^{2}(s, x) d s
$$

Denoting by $\left(\xi_{t}, \Pi_{x}\right)$ a Brownian motion started from time zero and coordinate $x$, Eq.(17) may be rewritten

$$
\begin{aligned}
v(t, x) & =\Pi_{x}\left\{e^{-t} g\left(\xi_{t}\right)+\int_{0}^{t} e^{-(t-s)} v^{2}\left(s, \xi_{t-s}\right) d s\right\} \\
& =\Pi_{x}\left\{e^{-t} g\left(\xi_{t}\right)+\int_{0}^{t} e^{-s} v^{2}\left(t-s, \xi_{s}\right) d s\right\}
\end{aligned}
$$

Therefore the solution is obtained by the following process:

At the initial time, a single particle begins a Brownian motion, starting from $x$ and continuing for an exponential holding time $T$ with $P(T>t)=$ $e^{-t}$. 
Then, at $T$, the particle splits into two, the new particles continuing along independent Brownian paths starting from $x(T)$. These particles, in turn, are subjected to the same splitting rule, meaning that after an elapsed time $t>0$ one has $n$ particles located at $x_{1}(t), x_{2}(t), \cdots x_{n}(t)$ with $P(n=k)=$ $e^{-t}\left(1-e^{-t}\right)^{k-1}$.

The solution of (7) is obtained by

$$
v(t, x)=\mathbb{E}\left\{g\left(x_{1}(t)\right) g\left(x_{2}(t)\right) \cdots g\left(x_{n}(t)\right)\right\}
$$

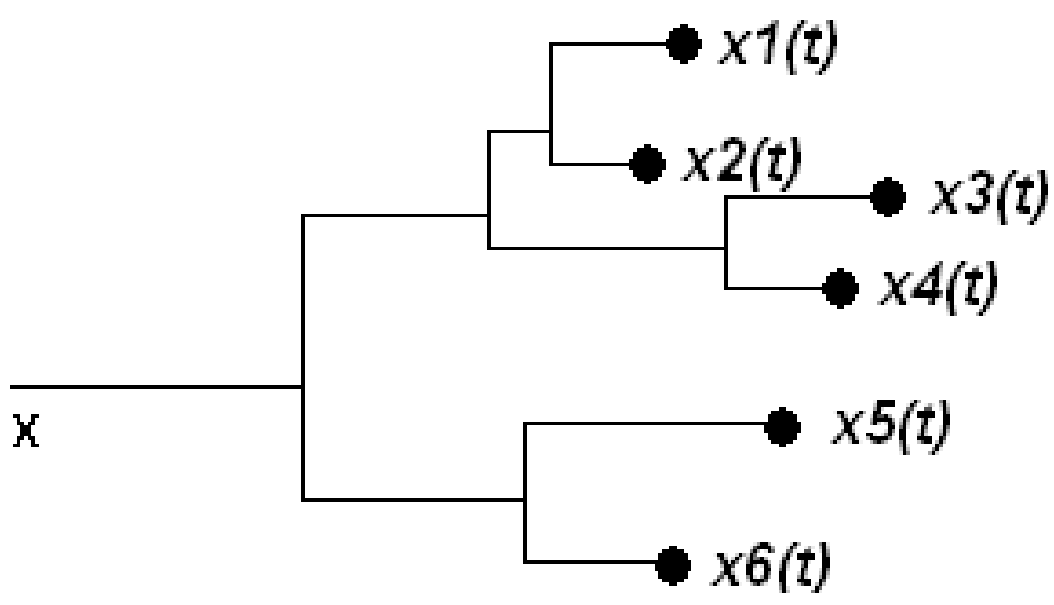

Figure 1: The McKean process for the KPP equation

An equivalent interpretation consists in considering the process propagating backwards in time from time $t$ at the point $x$ and, when it reaches time zero, it samples the initial condition. That is, the process generates a measure at the $t=0$ boundary which is then applied to the function $g(x)=v(0, x)$.

This construction, which expresses the solution as a stochastic multiplicative functional of the initial condition, is also qualitatively equivalent to importance sampling of the Picard iteration of Eq.(77).

A sufficient condition for the existence of (9) is $|g(x)| \leq 1$ or, almost surely, $|g(x)|\left(1-e^{-t}\right) \leq 1$.

Another probabilistic approach to this type of equations is through the construction of superprocesses. In many cases a superprocess may be looked at as the scaling limit of a branching particle system. The point of view used 
in the derivation of superprocesses is different from the derivation above. In the next subsection a short introduction to superprocesses is sketched and then the KPP solution is constructed via superprocesses.

\subsection{Branching exit measures and superprocesses}

Let $(E, \mathcal{B})$ be a measurable space and $M_{+}(E)$ the space of finite measures in $E$. Denote by $\left(X_{t}, P_{0, \mu}\right)$ a branching stochastic process with values in $M_{+}(E)$ and transition probability $P_{0, \mu}$ starting from time 0 and measure $\mu$. The process is said to satisfy the branching property if given $\mu=\mu_{1}+\mu_{2}$

$$
P_{0, \mu}=P_{0, \mu_{1}} * P_{0, \mu_{2}}
$$

that is, after the branching $\left(X_{t}^{1}, P_{0, \mu_{1}}\right)$ and $\left(X_{t}^{2}, P_{0, \mu_{2}}\right)$ are independent and $X_{t}^{1}+X_{t}^{2}$ has the same law as $\left(X_{t}, P_{r 0, \mu}\right)$. In terms of the transition operator $V_{t}$ operating on functions on $E$ this is

$$
V_{t} f\left(\mu_{1}+\mu_{2}\right)=V_{t} f\left(\mu_{1}\right)+V_{t} f\left(\mu_{2}\right)
$$

where $e^{-\left\langle V_{t} f, \mu\right\rangle} \stackrel{\circ}{=} P_{r, \mu} e^{-\left\langle f, X_{t}\right\rangle}$ or

$$
V_{t} f(\mu)=-\log P_{0, \mu} e^{-\left\langle f, X_{t}\right\rangle}
$$

$V_{t}$ is called the log-Laplace semigroup associated to $X_{t}$. In (12) if the initial measure $\mu$ is $\delta_{x}$ one writes

$$
V_{t} f(x)=-\log P_{0, x} e^{-\left\langle f, X_{t}\right\rangle}
$$

By (10) the probability law of $X_{t}$ is infinitely divisible.

Now in $S=[0, \infty) \times E$ consider a set $Q \subset S$ and the associated branching exit process $\left(X_{Q}, P_{\mu}\right)$ composed of a propagating Markov process in $E$, $\xi=\left(\xi_{t}, \Pi_{0, x}\right)$, a set of probabilities $p_{n}(t, x)$ describing the branching and a parameter $k$ defining the lifetime.

$$
u(x)=V_{Q} f(x)=-\log P_{0, x} e^{-\left\langle f, X_{Q}\right\rangle}
$$

$\left\langle f, X_{Q}\right\rangle$ is the integral of the function $f$ on the (space-time) boundary with the boundary exit measure generated by the process. One says that this branching exit process is a $(\xi, \psi)$-superprocess if $u(x)$ satisfies the equation

$$
u+G_{Q} \psi(u)=K_{Q} f
$$


where $G_{Q}$ is the Green operator,

$$
G_{Q} f(r, x)=\Pi_{0, x} \int_{0}^{\tau} f\left(s, \xi_{s}\right) d s
$$

$K_{Q}$ the Poisson operator

$$
K_{Q} f(x)=\Pi_{0, x} 1_{\tau<\infty} f\left(\xi_{\tau}\right)
$$

$\psi(u)$ means $\psi(0, x ; u(0, x))$ and $\tau$ is the exit time from $Q$.

The superprocess is constructed as follows: Let $\varphi(s, x ; z)$ be the offspring generating function at time $s$ and point $x$

$$
\varphi(s, x ; z)=c \sum_{0}^{\infty} p_{n}(s, x) z^{n}
$$

where $\sum_{n} p_{n}=1$ and $c$ denotes the branching intensity.

Then for $e^{-w(0, x)} \doteq P_{0, x} e^{-\left\langle f, X_{Q}\right\rangle}$ one has

$$
P_{0, x} e^{-\left\langle f, X_{Q}\right\rangle} \stackrel{\circ}{=} e^{-w(0, x)}=\Pi_{0, x}\left[e^{-k \tau} e^{-f\left(\tau, \xi_{\tau}\right)}+\int_{0}^{\tau} d s k e^{-k s} \varphi\left(s, \xi_{s} ; e^{-w\left(\tau-s, \xi_{s}\right)}\right)\right]
$$

The measure-valued process starts from $\delta_{x}$ at time $0, \tau$ is the first exit time from $Q$ and $f\left(\tau, \xi_{\tau}\right)$ the value of a function in the boundary $\partial Q$.

Using $\int_{0}^{\tau} k e^{-k s} d s=1-e^{-k \tau}$ and the Markov property $\Pi_{0, x} 1_{s<\tau} \Pi_{s, \xi_{s}}=$ $\Pi_{0, x} 1_{s<\tau}$, Eq.(19) for $e^{-w(0, x)}$ is converted into

$$
e^{-w(0, x)}=\Pi_{0, x}\left[e^{-f\left(\tau, \xi_{\tau}\right)}+k \int_{0}^{\tau} d s\left[\varphi\left(s, \xi_{s} ; e^{-w\left(\tau-s, \xi_{s}\right)}\right)-e^{-w\left(\tau-s, \xi_{s}\right)}\right]\right]
$$

This is lemma 1.2 in ch.4 of Ref.[5]. Because of the central role of this result for the construction of superprocesses, a proof is included in the Appendix with the notations used in this paper.

Eq.(2.11) is now obtained by a limiting process. Let in (20) replace $w(0, x)$ by $\beta w_{\beta}(0, x)$ and $f$ by $\beta f . \quad \beta$ is interpreted as the mass of the particles and when the measure-valued process $X_{Q} \rightarrow \beta X_{Q}$ then $P_{\mu} \rightarrow P_{\frac{\mu}{\beta}}$.

$e^{-\beta w(0, x)}=\Pi_{0, x}\left[e^{-\beta f\left(\tau, \xi_{\tau}\right)}+k_{\beta} \int_{0}^{\tau} d s\left[\varphi_{\beta}\left(s, \xi_{s} ; e^{-\beta w\left(\tau-s, \xi_{s}\right)}\right)-e^{-\beta w\left(\tau-s, \xi_{s}\right)}\right]\right]$ 
Defining

$$
u_{\beta}=\left(1-e^{-\beta w_{\beta}}\right) / \beta \quad ; \quad f_{\beta}=\left(1-e^{-\beta f}\right) / \beta
$$

and

$$
\psi_{\beta}\left(r, x ; u_{\beta}\right)=\frac{k_{\beta}}{\beta}\left(\varphi\left(r, x ; 1-\beta u_{\beta}\right)-1+\beta u_{\beta}\right)
$$

one obtains from (21)

$$
u_{\beta}(0, x)+\Pi_{0, x} \int_{0}^{\tau} d s \psi_{\beta}\left(s, \xi_{s} ; u_{\beta}\right)=\Pi_{0, x} f_{\beta}\left(\tau, \xi_{\tau}\right)
$$

that is

$$
u_{\beta}+G_{Q} \psi_{\beta}\left(u_{\beta}\right)=K_{Q} f_{\beta}
$$

When $\beta \rightarrow 0, f \rightarrow f_{\beta}$ and if $\psi_{\beta}$ goes to a well defined limit $\psi$ then $u_{\beta}$ tends to a limit $u$ solution of (15) associated to a superprocess. Also one sees from (22) that in the $\beta \rightarrow 0$ limit

$$
u_{\beta} \rightarrow w_{\beta}=-\log P_{0, x} e^{-\left\langle f, X_{Q}\right\rangle}
$$

as in Eq.(14). The superprocess corresponds to a cloud of particles for which both the mass and the lifetime tend to zero.

\subsection{The KPP equation as a superprocess}

When the integral Eq.(7) is interpreted probabilistically, it may be identified with Eq.(19) with $k=1, e^{-w(0, x)}=v(\tau, x), e^{-f\left(\tau, \xi_{\tau}\right)}=g\left(\xi_{\tau}\right), \varphi\left(s, \xi_{s} ; e^{-w\left(\tau-s, \xi_{s}\right)}\right)=$ $v^{2}\left(\tau-s, \xi_{s}\right)$. Therefore the McKean probabilistic construction corresponds to an intermediate step in the superprocess construction. At this level the process that is considered in Eq.(19) is the same as in McKean's construction. Summing over the exit measure, the solution is

$$
v(t, x)=e^{-\left\langle f, X_{Q}\right\rangle}=e^{-\sum_{i} f\left(\xi_{\tau_{i}}\right)}=e^{\sum_{i} \log g\left(\xi_{\tau_{i}}\right)}=\Pi_{i} g\left(\xi_{\tau_{i}}\right)
$$

essentially the same as in (9). However, there are two differences. First, the initial condition $g$ must be positive to have a well-defined logarithm. This is a restriction as compared to McKean's construction. But, on the other hand, the interpretation as an exit measure, allows to deal with Cauchy problems with boundary conditions. The exit measure is from the set $Q=[0, t] \times \Omega$, $\tau$ being the time at which the $\left(t, \xi_{t}\right)$ process reaches $\partial \Omega$ or $\tau=t$ inside $\Omega$. 
For the superprocess, let $u(t, x)=1-v(t, x)$, which satisfies the equation

$$
\frac{\partial u}{\partial t}=\frac{1}{2} \frac{\partial^{2} u}{\partial x^{2}}-u^{2}+u
$$

and the integral equation

$$
u(t, x)=G(t, x)(1-g(x))+\int_{0}^{t} G(s, x)\left(u(t-s, x)-u^{2}(t-s, x)\right) d s
$$

or

$$
u(t, x)+\Pi_{x} \int_{0}^{t}\left(u^{2}\left(t-s, \xi_{s}\right)-u\left(t-s, \xi_{s}\right)\right) d s=\Pi_{x}\left(1-g\left(\xi_{t}\right)\right)
$$

that is for KPP

$$
\psi(0, x ; u)=u^{2}-u
$$

Equating with (23) one obtains

$$
\begin{aligned}
\psi_{\beta}\left(0, x ; u_{\beta}\right) & =\frac{k_{\beta}}{\beta}\left(\varphi\left(0, x ; 1-\beta u_{\beta}\right)-1+\beta u_{\beta}\right) \\
& =\frac{k_{\beta}}{\beta}\left(c \sum p_{n}\left(1-\beta u_{\beta}\right)^{n}-1+\beta u_{\beta}\right) \\
& =\frac{k_{\beta} c}{\beta}\left(\beta^{2} u_{\beta}^{2}-\beta u_{\beta}\right) \\
& =u^{2}-u
\end{aligned}
$$

with $p_{n}=\delta_{n, 2}$. Therefore $c=\beta=1$ and $k_{\beta}=1$. That is, for KPP the superprocess is not a scaling limit. It coincides with the McKean process. However in this case, because $\beta=1$ instead of $\beta \rightarrow 0$, the solution is given by $\left(1-e^{-w}\right)$ instead of (14).

However, the power of the superprocesses is that, with other limiting choices of $\beta$, stochastic solutions may be constructed for other equations, in particular for solutions without the natural Poisson clock provided by the term $-v$ which is present in the KPP equation. For example for

$$
\frac{\partial v}{\partial t}=\frac{1}{2} \frac{\partial^{2} v}{\partial x^{2}}+v^{2}
$$

with $u=-v$

$$
\frac{\partial u}{\partial t}=\frac{1}{2} \frac{\partial^{2} u}{\partial x^{2}}-u^{2}
$$


one has

$$
\psi(0, x ; u)=u^{2}
$$

which equated with (23)

$$
\begin{aligned}
\psi_{\beta}\left(0, x ; u_{\beta}\right) & =\frac{k_{\beta}}{\beta}\left(\beta u_{\beta}-1+\sum_{n=0}^{2} p_{0}+p_{1}\left(1-\beta u_{\beta}\right)+p_{2}\left(1-\beta u_{\beta}\right)^{2}\right) \\
& =u_{\beta}^{2}
\end{aligned}
$$

leads to

$$
p_{1}=0 ; \quad p_{0}=p_{2}=\frac{1}{2} ; \quad k_{\beta}=\frac{2}{\beta}
$$

In this case one may let $\beta \rightarrow 0$, the solution is given by (14) and the superprocess corresponds to the scaling limit $(n \rightarrow \infty$ in Fig.2) of a process where both the mass and the lifetime of the particles tends to zero and at each bifurcation point one has equal probability of either dying without offspring or having two children (Fig.2)

This construction may be generalized for interactions $u^{\alpha}$ with $1<\alpha \leq 2$. With $z=1-\beta u_{\beta}$ one has

$$
\begin{aligned}
\varphi(0, x ; z) & =\sum_{n} p_{n} z^{n}=z+\frac{\beta}{k_{\beta}} u_{\beta}^{\alpha}=z+\frac{\beta}{k_{\beta}} \frac{(1-z)^{\alpha}}{\beta^{\alpha}} \\
& =z+\frac{1}{k_{\beta} \beta^{\alpha-1}}\left(1-\alpha z+\frac{\alpha(\alpha-1)}{2} z^{2}-\frac{\alpha(\alpha-1)(\alpha-2)}{3 !} z^{3}+\cdot(\cdot 38)\right)
\end{aligned}
$$

Choosing $k_{\beta}=\frac{\alpha}{\beta^{\alpha-1}}$ the terms in $z$ cancel and for $1<\alpha \leq 2$ the coefficients of all the remaining $z$ powers are positive and may be interpreted as branching probabilities. It would not be so for $\alpha>2$. Then

$$
p_{0}=\frac{1}{\alpha} ; \quad p_{1}=0 ; \quad \cdots \quad p_{n}=\frac{(-1)^{n}}{\alpha}\left(\begin{array}{c}
\alpha \\
n
\end{array}\right) \quad n \geq 2
$$

with $\sum_{n} p_{n}=1$. With this choice of branching probabilities, $k_{\beta}=\frac{\alpha}{\beta^{\alpha-1}}$ and $\beta \rightarrow 0$ one obtains a superprocess which, through (14), provides a solution to the equation

$$
\frac{\partial u}{\partial t}=\frac{1}{2} \frac{\partial^{2} u}{\partial x^{2}}-u^{\alpha}
$$

for $1<\alpha \leq 2$.

However, the superprocess cannot be constructed for $\alpha>2$ because some of the $z^{n}$ coefficients in the offspring generating function $\varphi(0, x ; z)$ would be negative. 


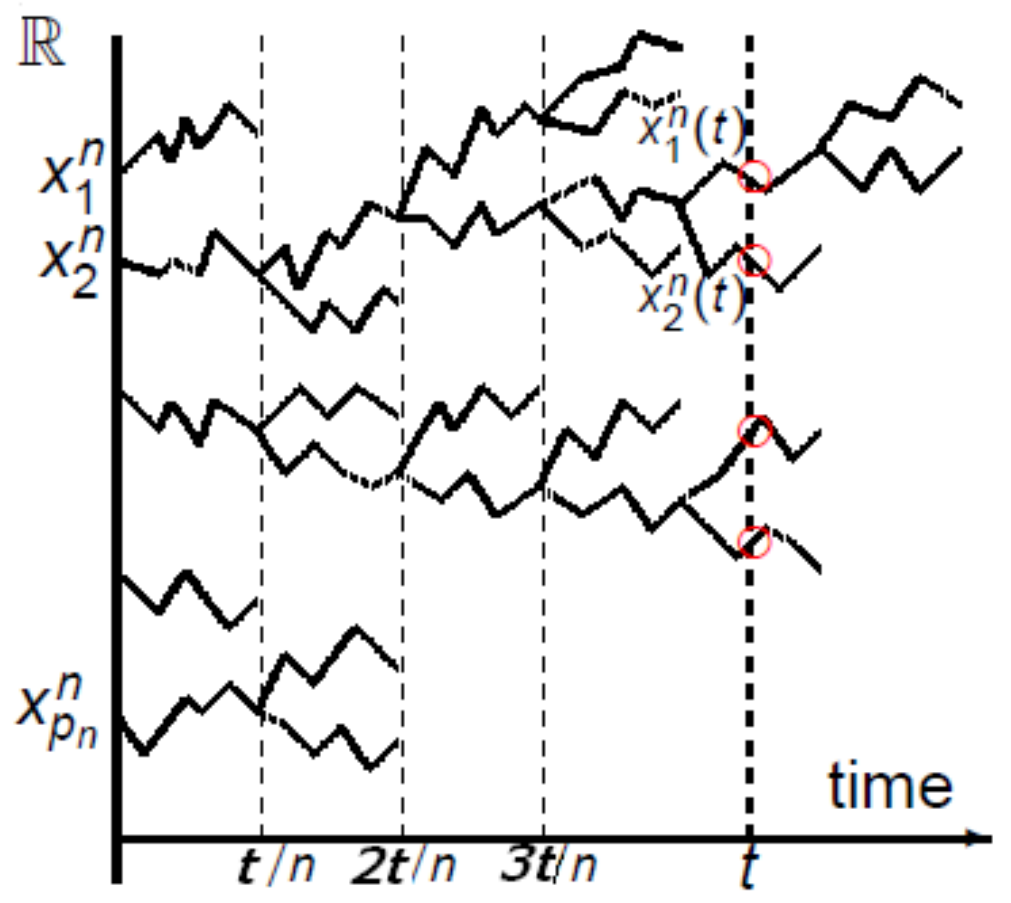

Figure 2: The branching process which in the scaling limit $n \rightarrow \infty$ leads to the superprocess solution of Eq.(34)

\section{Appendix: Proof of the lemma}

Let

$$
u(x, t)=\Pi_{0, x}\left\{e^{-k t} u\left(\xi_{t}, 0\right)+\int_{0}^{t} k e^{-k s} \Phi\left(\xi_{s}, t-s\right) d s\right\}
$$

Then

$$
\begin{aligned}
\Pi_{0, x} \int_{0}^{t} k u\left(\xi_{s}, t-s\right) d s= & \Pi_{0, x}\left\{\int_{0}^{t} k e^{-k(t-s)} u\left(\xi_{s+t-s}, 0\right) d s\right. \\
& \left.+\int_{0}^{t} k d s \int_{0}^{t-s} k d s^{\prime} e^{-k s^{\prime}} \Phi\left(\xi_{s+s^{\prime}}, t-s-s^{\prime}\right)\right\}
\end{aligned}
$$


Summing (41) and (42)

$$
\begin{aligned}
& u(x, t)+\Pi_{0, x} \int_{0}^{t} k u\left(\xi_{s}, t-s\right) d s \\
= & \Pi_{0, x}\left\{\left(e^{-k t}+\int_{0}^{t} k e^{-k(t-s)} d s\right) u\left(\xi_{t}, 0\right)\right. \\
& \left.+k \int_{0}^{t} e^{-k s} \Phi\left(\xi_{s}, t-s\right) d s+k \int_{0}^{t} d s \int_{0}^{t-s} k d s^{\prime} e^{-k s^{\prime}} \Phi\left(\xi_{s+s^{\prime}}, t-s-s^{\prime}\right) d s^{\prime}\right\}
\end{aligned}
$$

Changing variables in the last integral in (43) from $\left(s, s^{\prime}\right)$ to $\left(s, \sigma=s+s^{\prime}\right)$ one obtains for the last term

$$
k \int_{0}^{t} d \sigma \int_{0}^{\sigma} k d s e^{-k(\sigma-s)} \Phi\left(\xi_{\sigma}, t-\sigma\right) d s
$$

and finally

$$
\begin{aligned}
& u(x, t)+\Pi_{0, x} k \int_{0}^{t} u\left(\xi_{s}, t-s\right) d s \\
= & \Pi_{0, x}\left\{u\left(\xi_{t}, 0\right)+k \int_{0}^{t} \Phi\left(\xi_{s}, t-s\right) d s\right\}
\end{aligned}
$$

\section{References}

[1] R. M. Blumenthal and R. K. Getoor; Markov processes and potential theory, Academic Press, New York 1968.

[2] R. F. Bass; Probabilistic techniques in analysis, Springer, New York 1995.

[3] R. F. Bass; Diffusions and elliptic operators, Springer, New York 1998.

[4] H. P. McKean; Comm. on Pure and Appl. Math. 28 (1975) 323-331, 29 (1976) 553-554.

[5] E. B. Dynkin; Diffusions, Superdiffusions and Partial Differential Equations, AMS Colloquium Pubs., Providence 2002. 
[6] E. B.Dynkin; Superdiffusions and positive solutions of nonlinear partial differential equations, AMS, Providence.2004.

[7] Y. LeJan and A. S. Sznitman ; Prob. Theory and Relat. Fields 109 (1997) 343-366.

[8] E. C. Waymire; Prob. Surveys 2 (2005) 1-32.

[9] R. N. Bhattacharya et al. ; Trans. Amer. Math. Soc. 355 (2003) 50035040

[10] M. Ossiander ; Prob. Theory and Relat. Fields 133 (2005) 267-298.

[11] J. C. Orum; Stochastic cascades and 2D Fourier Navier-Stokes equations, in Lectures on multiscale and multiplicative processes, www.maphysto.dk/publications/MPS-LN/2002/11.pdf

[12] R. Vilela Mendes and F. Cipriano; Commun. Nonlinear Science and Num. Simul. 13 (2008) 221-226 and 1736.

[13] E. Floriani, R. Lima and R. Vilela Mendes; European Physical Journal D 46 (2008) 295-302 and 407.

[14] R. Vilela Mendes; Stochastics 81 (2009) 279-297.

[15] R. Vilela Mendes; J. Math. Phys. 51 (2010) 043101.

[16] F. Cipriano, H. Ouerdiane and R. Vilela Mendes; Fract. Calc. Appl. Anal. 12 (2009) 47-56.

[17] J. A. Acebrón, A. Rodriguez-Rozas and R. Spigler; J. of Comp. Physics 228 (2009) 5574-5591.

[18] J.A. Acebrón, A. Rodríguez-Rozas and R. Spigler; J. on Scientific Computing 43 (2010) 135-157.

[19] J.A. Acebrón and A. Rodríguez-Rozas; J. Comp. Phys. 230 (2011) 78917909. 